CERN-TH/95-81

hep-th/9503235

\title{
Loop scattering in two-dimensional QCD
}

\author{
E. Abdalla ${ }^{(1),(2) *}$ and M.C.B. Abdalla ${ }^{(1) \dagger}$ \\ (1) CERN-TH \\ CH-1211 Geneva 23 - Switzerland \\ (2) Institut für Theoretische Physik, Universität Heidelberg \\ Philosophenweg 16, 6900 Heidelberg 1- Germany
}

\begin{abstract}
Using the integrability conditions that we recently obtained in $\mathrm{QCD}_{2}$ with massless fermions, we arrive at a sufficient number of conservation laws to be able to fix the scattering amplitudes involving a local version of the Wilson loop operator.
\end{abstract}

CERN-TH/95-81

hep-th/9503235

March 1995

* Permanent address: Instituto de Física - USP, C.P. 20516, S. Paulo, Brazil.

$\dagger$ Permanent address: Instituto de Física Teórica - UNESP, R. Pamplona 145, 01405-000, S. Paulo, Brazil. 
Two-dimensional QCD has been studied by several authors (see ref. [1] for a review). The theory presents, in a clear way, several features expected to characterize strong interactions, but besides such desirable physical features, it is possible to understand several of its properties analytically. In fact, we claim that it may be possible to obtain its full on-shell solution, as far as the scattering of closed loop (white) operators is concerned.

We represent the fermionic determinant in terms of a bosonic functional integral over the (exponential of $i$ times the) WZW action, ${ }^{2,3}$ and use the Polyakov-Wiegmann identity ${ }^{2}$ to split the action of the product of two fields in terms of the WZW action of each single field and a contact term; this shows that, at the Lagrangian level, the dynamics factorizes in terms of several conformally invariant theories (WZW and ghost actions), and a non-conformally invariant piece, given by a WZW action perturbed off criticality, which contains the (main) dynamical information. Finally, these fields, decoupled at the Lagrangian level, are coupled via BRST constraints ${ }^{4}$ obeyed by the theory, implying that only white objects appear in the physical subspace. The most important result implying the possibility of computing the $S$-matrix is the integrability of the afore-mentioned off-critically perturbed WZW theory. ${ }^{5}$

Let us briefly summarize the known results. The two-dimensional QCD partition function with massless quarks is given by

$$
\begin{aligned}
\mathcal{Z}\left[i_{\mu}, \eta, \bar{\eta}\right]= & \int \mathcal{D} \psi \mathcal{D} \bar{\psi} \mathcal{D} A_{\mu} \mathcal{D} E \mathcal{D}[\text { ghosts }] \\
& \times \mathrm{e}^{i S_{\text {ghosts }}+i \int \mathrm{d}^{2} x\left[\bar{\psi} i \not \mathcal{} \psi-\frac{1}{2} \operatorname{tr} E^{2}+\frac{1}{2} \operatorname{tr} E F_{+-}+i^{\mu} A_{\mu}+\bar{\eta} \psi+\bar{\psi} \eta\right]} .
\end{aligned}
$$

The fermion may be integrated out in terms of the gauge potentials $U$ and $V$, introduced as

$$
A_{+}=\frac{i}{e} U^{-1} \partial_{+} U \quad, \quad A_{-}=\frac{i}{e} V \partial_{-} V^{-1} ;
$$

these in turn, are implemented in the quantum case via inclusion of the corresponding Jacobian in (1). As a result one obtains the WZW action upon use of

$$
\operatorname{det} i \not D=\mathrm{e}^{i \Gamma[U V]}
$$

and

$$
\operatorname{det}\left(\frac{\partial A_{+}}{\partial U} \frac{\partial A_{-}}{\partial V}\right)=\operatorname{det} i \not D^{\text {adj }}=\mathrm{e}^{i c_{V} \Gamma[U V]},
$$

where a gauge-invariant regularization prescription has been chosen to evaluate the Jacobian, fixing the contact term $A_{+} A_{-}$; therefore, the result is a function of the gaugeinvariant product $U V$; the well-known WZW functional reads

$$
\Gamma[U]=\frac{1}{8 \pi} \int \mathrm{d}^{2} x \partial^{\mu} U^{-1} \partial_{\mu} U+\frac{1}{4 \pi} \int_{0}^{1} \mathrm{~d} r \int \mathrm{d}^{2} x \epsilon^{\mu \nu} \widetilde{U}^{-1} \dot{\widetilde{U}} \widetilde{U}^{-1} \partial_{\mu} \widetilde{U} \widetilde{U}^{-1} \partial_{\nu} \widetilde{U}
$$

obeying the Polyakov-Wiegmann identity

$$
\Gamma[U V]=\Gamma[U]+\Gamma[V]+\frac{1}{4 \pi} \operatorname{tr} \int \mathrm{d}^{2} x U^{-1} \partial_{+} U V \partial_{-} V^{-1} .
$$


In terms of the gauge-invariant field $\Sigma=U V$, the auxiliary $E$-field interaction becomes, upon introduction of $F_{+-}$as a function of $U$ and $V$,

$$
\operatorname{tr} E F_{+-}=\frac{i}{e} \operatorname{tr} U E U^{-1} \partial_{+}\left(\Sigma \partial_{-} \Sigma^{-1}\right)=\frac{i}{e} \operatorname{tr} \widetilde{E} \partial_{+}\left(\Sigma \partial_{-} \Sigma^{-1}\right)
$$

We change the auxiliary variable as

$$
\partial_{+} \widetilde{E}=\frac{i e\left(c_{V}+1\right)}{2 \pi} \beta^{-1} \partial_{+} \beta
$$

and use again the Polyakov-Wiegmann identity. This procedure leads to a complete separation of variables at the Lagrangian level, as we see from

$$
\begin{aligned}
& \mathcal{Z}\left[i_{\mu}, \eta, \bar{\eta}\right]=\int \mathcal{D} \widetilde{g} \mathrm{e}^{i \Gamma[\tilde{g}]} \mathcal{D} U \mathcal{D}[\text { ghosts }] \mathrm{e}^{i S_{\text {ghosts }}} \int \mathcal{D} \widetilde{\Sigma} \mathrm{e}^{-i\left(c_{V}+1\right) \Gamma[\tilde{\Sigma}]} \\
& \times \int \mathcal{D} \beta \mathrm{e}^{i \Gamma[\beta]+\frac{\mu^{2} i}{2} \operatorname{tr} \int \mathrm{d}^{2} z\left[\partial_{+}^{-1}\left(\beta^{-1} \partial_{+} \beta\right)\right]^{2}} \mathrm{e}^{i \int \mathrm{d}^{2} z i_{\mu} A_{\mu}-i \int \mathrm{d}^{2} z \mathrm{~d}^{2} w \bar{\eta}(z)(i \not D)^{-1}(z, w) \eta(w)},
\end{aligned}
$$

where $\tilde{g}=U g V, \widetilde{\Sigma}=\beta \Sigma$ and $\mu=\left(c_{V}+1\right) e / 2 \pi$. The quadratic and non-local piece, which we symbolize by $\Delta$, can be linearized and made local by the introduction of the identity

$$
\mathrm{e}^{\frac{i}{2} \mu^{2} \Delta}=\int \mathcal{D} C_{-} \mathrm{e}^{i \int \mathrm{d}^{2} x \frac{1}{2} \operatorname{tr}\left(\partial_{+} C_{-}\right)^{2}-\mu \operatorname{tr} \int \mathrm{d}^{2} x C_{-}\left(\beta^{-1} \partial_{+} \beta\right)},
$$

where $C_{-}$plays the role of an auxiliary field. The theory contains hidden constraints, as discussed elsewhere. ${ }^{4,5}$ Such constraints are essential to build the asymptotic states of the theory, which cannot have colour.

The non-trivial dynamical content of the theory is described by the $\beta$ field, which is the only non-conformally invariant piece of the theory. It is useful to discuss the model in parallel to its dual, obtained by rewriting the field $C_{-}$appearing in eq. (8) as $C_{-}=$ $\frac{i}{4 \pi \mu} W \partial_{-} W^{-1}$. After a simple change of variables and use of $(4 b)$, one obtains for the $\beta, C_{-}$integration the following partition function (below, we use $\widetilde{\beta}=\beta W$ ):

$$
\mathcal{Z}=\int \mathcal{D} \tilde{\beta} \mathrm{e}^{i \Gamma[\widetilde{\beta}]} \int \mathcal{D} W \mathrm{e}^{-i\left(c_{V}+1\right) \Gamma[W]-\frac{i}{2(4 \pi \mu)^{2}} \operatorname{tr} \int \mathrm{d}^{2} z\left[\partial_{+}\left(W \partial_{-} W^{-1}\right)\right]^{2}},
$$

or equivalently, the dual action

$$
S=-\left(c_{V}+1\right) \Gamma[W]+\operatorname{tr} \frac{1}{2} \int \mathrm{d}^{2} x\left(-B^{2}+\frac{1}{2 \pi \mu} \partial_{+} B \partial_{-} W W^{-1}\right),
$$

where the field $B$ plays, in the dual case, the same role as $C_{-}$did before, namely it is an auxiliary field. The theory (7) and its dual (9) can be studied by simple methods. The $\beta$ (resp. $W$ ) equations of motion correspond to a conservation law

$$
\partial_{+}\left(I_{-}^{\beta, W}\right)=0,
$$

where 


$$
\begin{gathered}
I_{-}^{\beta}\left(x^{-}\right)=4 \pi \mu^{2} J_{-}^{\beta}\left(x^{+}, x^{-}\right)+\partial_{+} \partial_{-} J_{-}^{\beta}\left(x^{+}, x^{-}\right)+\left[J_{-}^{\beta}\left(x^{+}, x^{-}\right), \partial_{+} J_{-}^{\beta}\left(x^{+}, x^{-}\right)\right] \\
I_{-}^{W}\left(x^{-}\right)=\frac{\left(c_{V}+1\right)}{4 \pi} J_{-}^{W}+\frac{1}{(4 \pi \mu)^{2}} \partial_{+} \partial_{-} J_{-}^{W}+\frac{1}{(4 \pi \mu)^{2}}\left[J_{-}^{W}, \partial J_{-}^{W}\right] .
\end{gathered}
$$

Notice that for the current $I_{-}^{W}\left(x^{-}\right)$we have only local expressions. Concerning the current $I_{-}^{\beta}$, the first class constraints are given by

$$
\begin{aligned}
& i \widetilde{g} \partial_{-} \widetilde{g}^{-1}-i\left(c_{V}+1\right) \Sigma \partial_{-} \Sigma^{-1}+J_{-} \text {(ghosts) } \sim 0, \\
& i \widetilde{g}^{-1} \partial_{+} \widetilde{g}-i\left(c_{V}+1\right) \Sigma^{-1} \partial_{+} \Sigma+J_{+}(\text {ghosts }) \sim 0,
\end{aligned}
$$

leading to two BRST nilpotent charges $Q^{( \pm)}$, as discussed in refs. [4, 5]. Therefore we find two first-class constraints. For the dual theory one finds a very similar structure (see [1, 5]). Second class constraints also show up, being given by

$$
\begin{gathered}
\Omega_{i j}^{\beta}=\left(\beta \partial_{-} \beta^{-1}\right)_{i j}+4 i \pi \mu\left(\beta C_{-} \beta^{-1}\right)_{i j}-\left(\widetilde{g} \partial_{-} \widetilde{g}^{-1}\right)_{i j} \\
\Omega_{i j}^{W}=\left(c_{V}+1\right) \Sigma^{-1} \partial_{+} \Sigma-\left(c_{V}+1\right) W^{-1} \partial_{+} W+\frac{1}{\mu} W^{-1} \partial_{+} B W=0 .
\end{gathered}
$$

To obtain the consequences of such a huge set of conservation laws and constraints, we have to verify how the corresponding charges act on asymptotic states. This action can be unravelled once one knows the Lorentz transformation properties of the charges. A useful set of conserved charges is

$$
Q^{(n)}=\int \mathrm{d} x(t-x)^{n} I_{-}(x) .
$$

It is not difficult to obtain

$$
\left[T, Q^{(n)}\right]=n Q^{(n)},
$$

where the Lorentz generator $T$ acts on an asymptotic one-particle state as a derivative with respect to the rapidity $\theta$ :

$$
T=\frac{d}{d \theta}
$$

from which one obtains

$$
Q^{(n)} \simeq\left(p_{-}\right)^{n} J \quad,
$$

where $J$ is the generator of the left transformations for the $\beta$ fields. This would mean that the first charge, $Q^{(1)}$, is the generator of right- $S U(N)$ transformations for the $\beta$ fields! In the quantum theory there is no contribution from the short-distance expansion of $J_{-}$and $\partial_{\mu} J_{-}$, since divergences are too mild. The $S U(N)$ transformation generators are simple to compute. In the $\beta$ language one has, for left-SU(N) transformations:

$$
\begin{aligned}
& J_{+}^{L_{S U(N)}}=0 \\
& J_{-}^{L_{S U(N)}}=-\frac{1}{2 \pi} \partial_{-} \beta \beta^{-1}+i \mu \beta C_{-} \beta^{-1},
\end{aligned}
$$


while for right-SU(N) transformations we obtain

$$
\begin{aligned}
& J_{-}^{R_{S U(N)}}=i \mu C_{-}+\left[\partial_{+} C_{-}, C_{-}\right] \\
& J_{+}^{R_{S U(N)}}=-\frac{1}{2 \pi} \beta^{-1} \partial_{+} \beta
\end{aligned}
$$

For the first set, i.e. the left transformations, one finds that the currents are equivalent to analogous chiral currents corresponding to free fields. On the other hand, the right transformations lead to an infinite number of conservation laws due to the presence of the Lax pair, and reproduce eq. (10); therefore we have confirmed the fact that (10) generates the right- $S U(N)$ transformations. For the left transformations, we obtain only left-moving currents, which explain constraints such as (12), where they are expressed in terms of free WZW currents.

The structure is even clearer in the $W$ language, where the right- $S U(N)$ transformations are

$$
\begin{aligned}
& J_{+}^{R_{S U(N)}}=0 \\
& J_{-}^{R_{S U(N)}}=-\frac{c_{V}+1}{2 \pi} W^{-1} \partial_{+} W+\frac{1}{4 \pi \mu} W^{-1} \partial_{+} B W
\end{aligned}
$$

while the left-SU(N) transformations are

$$
\begin{aligned}
& J_{-}^{L_{S U(N)}}=\frac{c_{V}+1}{4 \pi} \partial_{-} W W^{-1}+\frac{1}{4 \pi \mu}\left[B, \partial_{-} W W^{-1}\right], \\
& J_{+}^{L_{S U(N)}}=-\frac{1}{4 \pi \mu} \partial_{+} B .
\end{aligned}
$$

In the $\beta_{a i}$ language the left indices, such as $a, b, c, d$ are thus free, described by a trivial $S$-matrix, while the right indices, such as $i, j, k, l$ are described by an $S U(N)$ covariant integrable $S$-matrix, which has a well-known classification. ${ }^{6}$ We thus write the (unique) $\beta$-ansatz as

$$
\begin{aligned}
\left\langle a i \theta_{1}, b j \theta_{2} \mid c k \theta_{3} d l \theta_{4}\right\rangle= & \delta\left(\theta_{1}-\theta_{3}\right) \delta\left(\theta_{2}-\theta_{4}\right) \delta_{a c} \delta_{b d}\left(\sigma_{1}(\theta) \delta_{i k} \delta_{j l}+\sigma_{2}(\theta) \delta_{i l} \delta_{j k}\right) \\
& +\delta\left(\theta_{1}-\theta_{4}\right) \delta\left(\theta_{2}-\theta_{3}\right) \delta_{a d} \delta_{b c}\left(\sigma_{1}(\theta) \delta_{i l} \delta_{j k}+\sigma_{2}(\theta) \delta_{i k} \delta_{j l}\right),
\end{aligned}
$$

where $\theta=\theta_{1}-\theta_{2}$ is the relative rapidity. A similar result for the dual theory exists, where the left/right definition has to be interchanged, namely the field is $W_{i a}$ in the dual case.

There are four classes of possible (non-trivial) $S$-matrices. Since the $\beta$ field represents a kind of Wilson-loop-type variable as given by (5), we suppose that, for large $N, \beta^{2} \sim \beta$, which implies an $S$-matrix of class II as classified in ref. [6], or $\sigma_{2}=\frac{2 \pi i}{N} \sigma_{1}$, and a bound state

$$
B_{N=\infty}(\theta)=\frac{\operatorname{sh}(\theta)+i \sin (\pi / 3)}{\operatorname{sh}(\theta)-i \sin (\pi / 3)} .
$$

Finally, the only asymptotic states compatible with gauge $S U(N)$ colour symmetry, are those confining such degrees of freedom; therefore we have the scattering elements ${ }^{7}$

$$
\left\langle\theta_{1} \theta_{2} \mid \theta_{3} \theta_{4}\right\rangle=B_{N}(\theta)\left[\delta\left(\theta_{1}-\theta_{3}\right) \delta\left(\theta_{2}-\theta_{4}\right)+\delta\left(\theta_{1}-\theta_{4}\right) \delta\left(\theta_{2}-\theta_{3}\right)\right]
$$


where, for large $N$, the amplitude is given by (21).

\section{Acknowledgements}

This work was partially supported by CAPES Brazil, by the World Laboratory, and the Alexander von Humboldt Stiftung.

\section{References}

[1] E. Abdalla and M.C.B. Abdalla, preprint CERN-TH/95-49, hep-th/9503002, to appear in Phys. Rep.

[2] A.M. Polyakov and P.B. Wiegmann, Phys. Lett. B 131 (1983) 121; B 141 (1984) 223

[3] E. Witten, Commun. Math. Phys. 92 (1984) 455

[4] D. Karabali and H.J. Schnitzer, Nucl. Phys. B 329 (1990) 649

[5] E. Abdalla and M.C.B. Abdalla, Phys. Lett. B 337 (1994) 347-353; and preprint CERN-TH/7354-94, hep-th/9407128, to appear in Int. J. Mod. Phys. A

[6] B. Berg, M. Karowski, V. Kurak, P. Weisz, Nucl. Phys. B 134 (1978) 125.

[7] E. Abdalla, M.C.B. Abdalla and K. Rothe, Non-perturbative methods in two-dimensional quantum field theory (World Scientific, Singapore, 1991) 